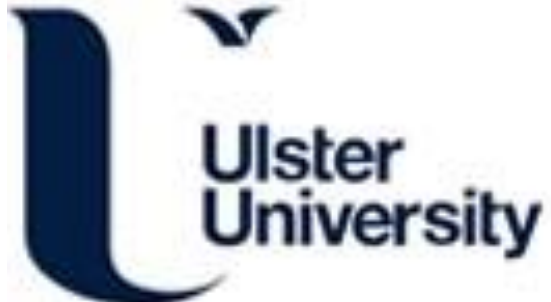

\section{Implementation of EEG based control of remote robotic systems}

Chakraborti, T., Sengupta, A., Banerjee, D., Konar, A., Bhattacharyya, S., Khasnobish, A., \& Janarthanan, R. (2011). Implementation of EEG based control of remote robotic systems. In 2011 International Conference on Recent Trends in Information Systems, ReTIS 2011 - Proceedings (pp. 203-208). [6146868] (2011 International Conference on Recent Trends in Information Systems, ReTIS 2011 - Proceedings). https://doi.org/10.1109/ReTIS.2011.6146868

Link to publication record in Ulster University Research Portal

Published in:

2011 International Conference on Recent Trends in Information Systems, ReTIS 2011 - Proceedings

Publication Status:

Published (in print/issue): 01/12/2011

DOI:

10.1109/ReTIS.2011.6146868

\section{Document Version}

Author Accepted version

\section{General rights}

Copyright for the publications made accessible via Ulster University's Research Portal is retained by the author(s) and / or other copyright owners and it is a condition of accessing these publications that users recognise and abide by the legal requirements associated with these rights.

\section{Take down policy}

The Research Portal is Ulster University's institutional repository that provides access to Ulster's research outputs. Every effort has been made to ensure that content in the Research Portal does not infringe any person's rights, or applicable UK laws. If you discover content in the Research Portal that you believe breaches copyright or violates any law, please contact pure-support@ulster.ac.uk. 


\section{Implementation of EEG Based Control of Remote Robotic Systems}

\author{
Tathagata Chakraborti ${ }^{1}$, Abhronil \\ Sengupta $^{2}$, Dhrubojyoti Banerjee ${ }^{3}$, \\ Amit Konar $^{4}$ \\ Department of Electronics and \\ Telecommunication Engineering \\ Jadavpur University \\ Kolkata, India \\ tathagata.net@live.com ${ }^{1}$ \\ senguptaabhronil@gmail.com ${ }^{2}$ \\ dhrubo_jyoti_banerjee@yahoo.co.in ${ }^{3}$ \\ konaramit@yahoo.co.in ${ }^{4}$
}

\author{
Saugat Bhattacharyya ${ }^{1}$, Anwesha \\ Khasnobish $^{2}$ \\ School of Bioscience and \\ Engineering \\ Jadavpur University \\ Kolkata, India \\ saugatbhattacharyya@gmail.com ${ }^{1}$ \\ anweshakhasno@gmail.com²
}

\author{
R. Janarthanan \\ Department IT \\ Jaya Engineering College \\ Thiruninravur, Chennai, India \\ srmjana_73@yahoo.com
}

\begin{abstract}
The paper provides a novel approach to control the motion and orientation of a mobile robot using an encoded sequence of arm movements, obtained from the motor imagery indicated by electroencephalographic measurements. The importance of the proposed scheme lies in maintaining secrecy and privacy in control or management of remote robotic systems, as the signals liberated from the user's brain is not accessible to any third party even during the execution phase of the command and hence can find applications in the defense sector. For our demonstration we have successfully differentiated six classes of limb movements from the raw EEG data, encoded the classified signals and used this to control the movements of a Khepera mobile robot. Experiments have been undertaken to study the suitability of the scheme, and the results are promising.
\end{abstract}

Keywords- BCI, EEG, Wavelet Transformation, PSD, RSVM, Khepera Mobile Robot-II.

\section{INTRODUCTION}

Recent developments in the field of Brain Computer Interfaces (BCIs) have opened up new possibilities of implementation in different fields like robotics, mass communication, automobiles, games, entertainment, and so on. EEG signals have been previously used to control rehabilitation arms in recovery of post stroke patients [1] and may be further used in similar situations in industries and warfare to control artificial limbs or exoskeletons or even robotic systems from remote locations. The use of EEG based control is becoming a growing area of research. Here we have demonstrated 'thought-control' of the motion of a robot by using EEG signals to control the movement of Khepera mobile robots. The fields in which such controlling schemes may be used are myriad. In medical research, EEG control is now getting popular for designing artificial limbs for people whose brains have lost their motor control to bypass the internal path between the brain and the muscles and provide the necessary control signals directly to the hand. In industry such control signals can be used to implement high precision work in diverse situations through robotic systems. In warfare, as mentioned before, a commander may choose to control robotic troops safely far away from the battlefield by using control signals generated from EEG since it possesses additional features of privacy and secrecy as the coded signals may not be readily intercepted. In recent years many such military robots have been developed like TALON, Predator or those made by iRobot to name a few and EEG may be used to control such systems remotely. Indeed the powered exoskeletons developed recently like the Sacros or the HULC will become many times more useful and convenient if EEG control can be implemented in them successfully.

The brain activities for BCI can be measured using EEG (electroencephalography). One of the main research areas of EEG based BCI for motor control is to decode the brain signals corresponding to particular limb movements [2]. A BCI system consists of modules to acquire brain signals, extract key features from them, and classify the features into intended classes ultimately aiming to translate into device commands. EEG based motor control study mainly relies on electrodes $\mathrm{C}_{3}$ and $\mathrm{C}_{4}$, as these lie on the scalp above the motor cortex area associated with voluntary motor control [13]. Various features like the time domain and frequency domain parameters [14-15], (STFT) [16], wavelet transforms, Spectral estimates [3], statistical parameters [17], Hjorth parameters [18], etc. are being used. Researchers have used various intelligent algorithms [4,11,17,19-22] viz. back propagation neural network, multilayer perceptron, discriminant analysis, support vector machines, vector quantization etc. to classify the EEG data in intended categories.

For controlling artificial systems in real life scenarios, it is essential to classify the left/right arm movement further into different joint movements. The more the degree of freedom, the more is the complexity of the systems that may be controlled because by combining different joint movements we can generate more complex coding schemes for extensive control. For left-right hand movement, the maximum classification accuracy of $87.50 \%$ is obtained using wavelet coefficient for RSVM classifier. For the multi-class classification, i.e. Finger-Elbow-Shoulder classification the maximum classification accuracy of $80.11 \%$ for elbow, 
$93.26 \%$ for finger and $81.12 \%$ for shoulder is obtained using the features obtained from power spectral density for RSVM classifier.

The following discussion essentially has three parts. First the methods of classification of the EEG signal are described. Next the control signals are developed with the help of a suitable coding scheme. Finally these signals are used to drive the Khepera robots and the accuracy of the method is discussed in the experimental results section. First we provide the block diagram of the proposed method below in Figure 1. The motor imagery as obtained from EEG measurements is classified into six classes of limb movements and sent to the Khepera robots through radio transmission after encryption using the codebook. The received signals are decoded at the receiver with the help of the codebook and the command signals for the Khepera are generated subsequently.

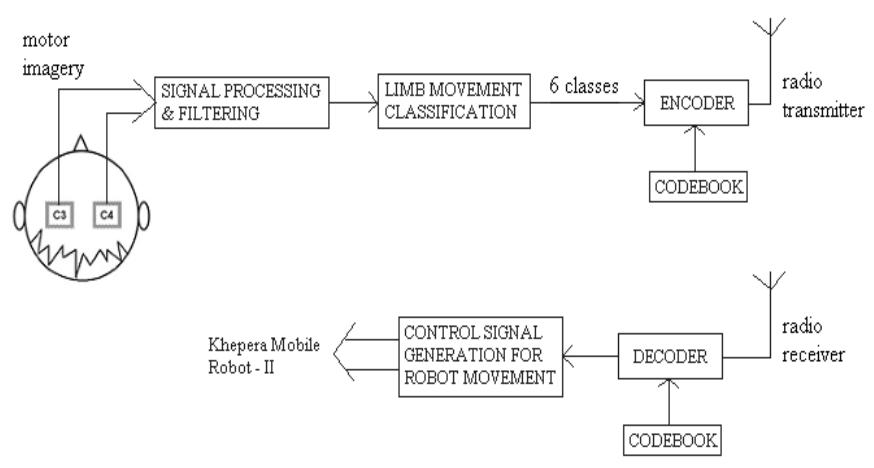

Figure 1. Motor imagery classification and coding for Khepera robot control through radio link

\section{PROPOSED WORK}

\section{A. Feature Extraction}

\section{1) Wavelet Transformation}

Wavelet transformation is a very effective way to extract features from a non-stationary signal like EEG [3, 4]. Their ability to discriminate both the temporal and spectral domain features of the signals makes them an important asset for EEG analysis. Also the wavelet transform do not suffer from the time-frequency trade off inherent in Short Time Fourier Transform (STFT) and Fourier Transform (FT) as their multiscale approximation allows for effective localization of the signal with various spectral-temporal characteristics. The discrete wavelet transforms analyzes the signals at different resolutions by decomposing the signal into coarse approximation and detail information. Each level comprises of two digital filters and two down-samplers by 2 . The downsampled outputs of the first high-pass and low-pass filters provides the detail D1 and approximation A1, respectively. The first approximation is further decomposed and the process continued, until the desired result is obtained $[5,6]$.

The wavelet transform is computed by convolution of the original signal $x(t)$ with a scaled and translated version of the mother wavelet function $\psi(t)$. The convolution leads to a new signal of wavelet coefficients,

$$
W_{x}^{\Psi}(a, b)=A \cdot \int_{-\infty}^{\infty} \Psi^{*}\left(\frac{t-b}{a}\right) \cdot x(t) \cdot d t
$$

where $\psi^{*}(t)$ represents the complex conjugate of the wavelet function, $b \in \mathbb{R}$ is the translation parameter, $a>0$ is the scaling parameter and $A=\frac{1}{\sqrt{a}}$ is the normalization parameter. The coefficients represent the similarity between the original signal and the wavelet function for particular values of $\mathrm{a}$ and $\mathrm{b}$. In the present study, Daubechies (db) mother wavelet of order 4 is used.

\section{2) Power Spectral Estimates}

Spectral density methods extract information from a signal to describe the distribution of its power in the frequency domain. The power spectral density (PSD) is defined as the Fourier transform (FT) of the signal's autocorrelation function, provided that the signal is stationary in a wide sense [5]. Thus for an EEG signal segmenting the complete time series data would be an ideal approach.

The measure for power spectral estimates is commonly divided into two methods: non-parametric method and parametric method. The Welch's method fall into nonparametric method which, divides the times series data into overlapping segments, computing a modified periodogram of each segment and then the PSD estimates are averaged. Let $x_{m}(n)=x(n+m N), n=0,1, \ldots, N-1$ denote the $\mathrm{m}^{\text {th }}$ block of the signal $\in C^{M N}$, with $\mathrm{M}$ denoting the number of blocks. Then the Welch PSD estimate is given by [12]:

$$
R_{x}\left(w_{k}\right)=\sum_{m=0}^{M-1}\left|D F T_{k}\left(x_{m}\right)\right|^{2}
$$

For this paper, the Welch approach was applied along with a Hamming window of length 125. The Welch method divides the times series data into overlapping segments, computing a modified periodogram of each segment and then the PSD estimates is averaged. The PSD estimates were obtained for two frequency bands, namely the alpha or mu band $(8-12 \mathrm{~Hz})$ and the central beta band $(18-25 \mathrm{~Hz})$ for each respective electrode. Also the average power was obtained for each band. Then the difference of the PSD estimates (3) and average power (4) is selected as another feature for this study.

$$
\begin{aligned}
& F_{P S D}=\sum_{f=a}^{b} P s d_{C 4}(f)-\sum_{f=a}^{b} P s d_{C 3}(f) \\
& F_{P O W}=\text { Pow }_{C 4}-\text { Pow }_{C 3}
\end{aligned}
$$

where, $a-b$ denotes the frequency range $(8-12 \mathrm{~Hz}$ for the alpha and $18-25 \mathrm{~Hz}$ for the beta bands), $P s d_{C 3 / C 4}$ is the PSD estimates of the respective electrodes in $[\mathrm{a}, \mathrm{b}], \mathrm{Pow}_{C 3 / C 4}$ is the average power of the respective electrodes in $[\mathrm{a}, \mathrm{b}]$.

\section{B. CLASSIFIER: RBF Support Vector Machine}

Statistical learning theory being the basis of support vector machines (SVM) provides a new approach to pattern recognition. Support vector machines (SVMs) are a set of related supervised learning methods used for classification and 
regression $[7,8]$. They belong to a family of generalized linear classifiers. SVM training always finds global minimum and its performance depends upon the selected kernel, where the user chooses only the error penalty parameter. The foundations of Support Vector Machines (SVM) have been developed by Vapnik [9] and gained popularity due to many promising features such as better empirical performance. The formulation uses the Structural Risk Minimization (SRM) principle, which has been shown to be superior, [10], to traditional Empirical Risk Minimization (ERM) principle, used by conventional neural networks. SRM minimizes an upper bound on the expected risk, where as ERM minimizes the error on the training data.

A support vector machine classifies by construction of a Ndimensional hyperplane that optimally separates the data in two categories. The separating hyperplane is specified only by the support vectors. As the complexity does not depend on the dimensionality of the feature space, SVM can deal with large feature spaces. A row of predictor values or a set of features that describes one case is called the vectors. Support vectors are the most important training points that define the hyperplane.

The linear classifier relies on dot product between vectors, $K\left(\boldsymbol{x}_{\boldsymbol{i}}, \boldsymbol{x}_{\boldsymbol{j}}\right)=\boldsymbol{x}_{\boldsymbol{i}}{ }^{T} \cdot \boldsymbol{x}_{\boldsymbol{j}}$ where $\boldsymbol{x}_{\boldsymbol{i}}$ and $\boldsymbol{x}_{\boldsymbol{j}}$ are the support vectors.

The training data is labeled as

$$
\left\{\mathbf{x}_{i}, \mathbf{x}_{j}\right\}, i=1, \ldots l, y_{i} \in\{-1,1\}, \mathbf{x}_{i} \in \mathbf{R}^{d}
$$

Suppose there is some hyperplane which separates the positive from the negative examples. The points $\boldsymbol{x}$ which lie on the hyperplane satisfy $\boldsymbol{w} \cdot \boldsymbol{x}+b=0$, where $\boldsymbol{w}$ is normal to the hyperplane, $|b| /|| w||$ is the perpendicular distance from the origin to the hyperplane, and $\|\boldsymbol{w}\|$ is the Euclidean norm of $\boldsymbol{w}$. Let $\boldsymbol{d}_{+}\left(\boldsymbol{d}_{-}\right)$be the shortest distance from the separating hyperplane to the closest positive (negative) example. The "margin" of a separating hyperplane is defined as $\boldsymbol{d}_{+}+\boldsymbol{d}_{-}$. The aim of linear support vector algorithm is to find the hyperplane with largest margin. Let us assume that all the training data satisfy the following constraints:

$$
\begin{aligned}
& \mathbf{x}_{\mathbf{i}} \cdot \mathbf{w}+b \geq+1 \text { for } \mathrm{y}_{i}=+1 \\
& \mathbf{x}_{\mathbf{i}} \cdot \mathbf{w}+b \leq-1 \text { for } \mathrm{y}_{i}=-1
\end{aligned}
$$

The above two equations can be combined to obtain the following resultant:

$$
\mathrm{y}_{i} \cdot\left(\mathbf{x}_{\mathbf{i}} \cdot \mathbf{w}+b\right)-1 \geq 0 \text { for all } i
$$

Considering the points for which the equality in (5) holds, these points lie on the hyperplane $\mathrm{H}_{1}: \boldsymbol{x}_{\boldsymbol{i}} \cdot \boldsymbol{w}+b=1$, where $\boldsymbol{w}$ is the normal and $|1-b| /|| \boldsymbol{w}||$ is the perpendicular distance from the origin. Similarly the points for which the equality in (6) holds lie on the hyperplane $\mathrm{H}_{2}: \boldsymbol{x}_{\boldsymbol{i}} \cdot \boldsymbol{w}+b=-1$. Hence $d_{+}=d_{-}=1 / \| w||$ and the margin is $d_{+}+d_{-}=2 /\|w\|$. Thus the problem is to minimize $\|\boldsymbol{w}\|^{2} / 2$ subject to condition (7).
When the vectors are separated by non-linear region, the SVM uses a kernel function to map the data into a different space where a hyperplane can be used for separating the vectors. Certain function that corresponds to an inner product in some expanded feature space is referred to as kernel function. According to Mercer's theorem, every semi positive definite symmetric function is a kernel. Kernel function transforms the data into higher dimensional space to make it possible for the separation of the vectors. The dot product becomes $K\left(\boldsymbol{x}_{\boldsymbol{i}}, \boldsymbol{x}_{\boldsymbol{j}}\right)=\varphi\left(\boldsymbol{x}_{\boldsymbol{i}}\right)^{T} \cdot \varphi\left(\boldsymbol{x}_{\boldsymbol{j}}\right)$ when every data point is mapped into high-dimensional space via some transformation $\Phi: x \rightarrow \varphi(x)$. The kernel matrix $K_{i j}=$ $K\left(\boldsymbol{x}_{i}, \boldsymbol{x}_{j}\right)$ is a Gram matrix in $H$ (i.e. the Euclidean space) [10]. It is necessary to choose $l$ training points such that the rank of the matrix $\mathrm{K}_{\mathrm{ij}}$ increases without limit as $l$ increases. The radial basis function is given by

$$
K\left(\boldsymbol{x}^{t}, \boldsymbol{x}\right)=\exp \left[-\frac{|| x^{t}-x||^{2}}{\sigma^{2}}\right]
$$

which defines a spherical kernel where $\boldsymbol{x}^{t}$ is the centre and $\sigma$, supplied by the user defines the radius.

\section{Developing the Control Signals}

Once the raw EEG signals have been classified with reasonable accuracy, they have been used to generate the necessary control signals as described below. The complexity of the task at hand (i.e. the number of different control signals needed) determine the kind of coding scheme used. In the current work, we have used the simplest case where each of the classified signals (say, right-hand-elbow or left-handfinger) are assigned a certain task and in this way we have six possible commands as given below:

TABLE I. CONTROL SIGNALS CORRESPONDING TO CLASSIFIED EEG SIGNALS

\begin{tabular}{|c|c|}
\hline EEG SIGNALS & CONTROL SIGNALS \\
\hline right elbow & move right \\
\hline right shoulder & move forward \\
\hline right finger & rotate clockwise \\
\hline left elbow & move left \\
\hline left shoulder & move backward \\
\hline left finger & rotate anticlockwise \\
\hline
\end{tabular}

In case of larger number of commands we need to combine a sequence of hand movements to generate a single control signal. For example, if we consider three consecutive time instants when the elbow-shoulder-finger movement is recorded, then we can generate a code with $2^{3}$ commands. In such case the user would need to execute, say for example, an elbow movement followed by two finger movements to execute forward movement of the robot. In this way more complex coding schemes may be developed. Even in cases of simple applications increasing the complexity is sometimes necessary to improve security otherwise the underlying coding scheme may be discovered through continuous observations 
whereas in cases of more complex schemes the control data becomes almost random in nature.

Once the coding scheme is complete, the subjects are provided with a 'code-book' that will provide the inverse relation of that provided by table I, i.e. it will tell the user which movements will produce which control commands. The hand movements and the commands which they translate into should ideally have some kind of logical relationship for the convenience of the user.

\section{EXPERIMENTAL RESULTS}

\section{A. Processing the EEG Data}

The experiment is so designed to make the subjects move their left or right hand along with moving a particular portion of the hand, namely, Finger, Elbow and Shoulder in a self paced manner.

\section{1) Experimental Setup}

Five females and three males (right handed) in the age group of $23 \pm 2$ years were employed as subjects in our experiment. The subjects were made to fill up a consent form and a simple introduction about the research work and stages of the experiment.

The experiment consists of 3 sessions with 20 trials each conducted on the same day with several minutes break in between. The subjects were asked to move the right and left hand, according to the visual cue displayed on the screen. In each session, the subjects were also asked to either move their finger, elbow or shoulder. In each trial, a blank screen was displayed in the first 2 seconds. In the $2^{\text {nd }}$ second a fixation cross ' + ' was displayed on the screen which indicates the beginning of the trial. From the 3 rd second onwards, the visual cue (left-right arrow) is displayed. At the same time, the subject was asked to move their respective limb according to the visual cue, until the display is blank again. The timing scheme of the visual cue is given below in Figure 2.

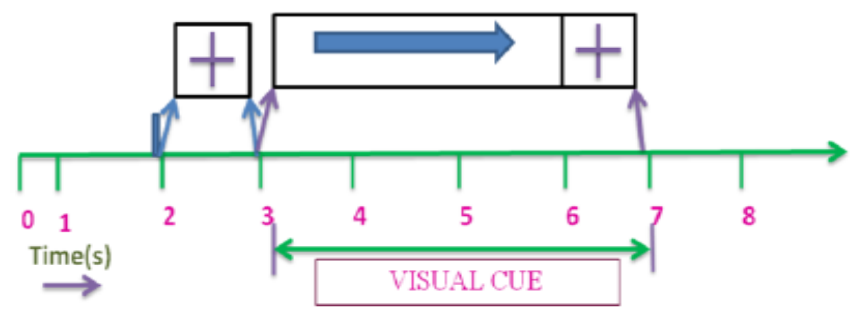

Figure 2. Timing scheme of the experiment

\section{2) Preprocessing}

For each subject a total of 60 trials were obtained of 8 second each. Out of the three electrodes used, C3 and C4 are selected for this study as these electrodes have greater relevance for extracting information on the left-right movement. Further, the obtained data was band-pass filtered using an elliptical filter (order 14) between 8 and $30 \mathrm{~Hz}$, for removing the noise based on the environment and recording techniques, and movement related information are mostly obtained in this bandwidth. The training and test data were selected randomly using the 10 fold cross validation technique which would be described later.

\section{3) Feature Extraction and Spectral Estimation Method}

As mentioned before here we have used Daubechies (db) mother wavelet of order 4. After trials with the EEG data, the D3 features and D4 features (Table II) i.e., the difference of the third and fourth level coefficient for the respective electrodes were selected as one of the feature components for the final feature vector (C4-C3).

The difference of the PSD estimates given by (1) and average power given by (2) is selected as another feature for this study as described previously.

TABLE II. EEG SIGNAL DECOMPOSITION INTO FREQUENCY BANDS WITH A SAMPLING FREQUENCY 0F $250 \mathrm{HZ}$

\begin{tabular}{|c|c|c|}
\hline $\begin{array}{c}\text { FREQUENCY } \\
\text { RANGE }\end{array}$ & $\begin{array}{c}\text { DECOMPOSITION } \\
\text { LEVEL }\end{array}$ & $\begin{array}{c}\text { FREQUENCY } \\
\text { BANDS }\end{array}$ \\
\hline $62.5-125$ & D1 & Noise \\
\hline $31.25-62.5$ & D2 & Gamma \\
\hline $15.625-31.25$ & D3 & Beta \\
\hline $7.8125-15.625$ & D4 & Alpha \\
\hline $3.91-7.8125$ & D5 & Delta \\
\hline
\end{tabular}

\section{4) Performance Analysis}

From the preprocessed filtered signal, only the data from the time interval $\mathrm{t}=3$ to $7 \mathrm{~s}$ are taken, marking the beginning and the end of the visual cue. From the two electrodes of interest, namely, $\mathrm{C} 3$ and $\mathrm{C} 4$, wavelet coefficients, PSD estimates for the alpha and beta bands and their corresponding powers were selected as the features for this study using the Wavelet toolbox and Signal Processing Toolbox in MATLAB. The classification ability of the obtained feature vectors can be measured through classification accuracy by averaging 10 times over a 10 fold cross validation. The 10 fold cross validation divides the number of samples into 10 disjoint sets, where 9 sets are for training and 1 set for testing. This procedure is repeated 10 times to obtain an average classification result. For our study, we have employed a twofold classification; i) left-right movement classification, and, ii) finger-elbow-shoulder movement classification. The average classification results are given in for 8 subjects using RSVM based approach. Since SVM is a binary class classifier, the one-against-all approach is employed for the elbow-fingershoulder classification, i.e., for example, this approach pits the elbow movement with the rest of the movement (fingershoulder movement) and the same for the rest of the movements. Table III and IV gives the classification results for left/right hand movement for their respective features, and Table V and VI gives the for elbow/finger/shoulder movement for the same. For left-right hand movement, the maximum classification accuracy of $87.50 \%$ is obtained using wavelet coefficient for RSVM classifier. Also by using PSD as the feature set, the maximum classification accuracy of $87.35 \%$ is obtained for RSVM classifier. 
TABLE III. RESULT OF LEFT/RIGHT CLASSIFICATION FOR WAVELET FEATURES

\begin{tabular}{|c|c|c|}
\hline SUBJECT ID & FEATURES & $\begin{array}{l}\text { CLASSIFICATION ACCURACY } \\
\text { (RSVM) }\end{array}$ \\
\hline 1 & \multirow{8}{*}{$\begin{array}{c}\text { Wavelet } \\
\text { (D3 \& D4 } \\
\text { Coeff.) }\end{array}$} & $.8615+/-.0621$ \\
\hline 2 & & $.7610+/-.0539$ \\
\hline 3 & & $.8697+/-.0593$ \\
\hline 4 & & $.5478+/-.0315$ \\
\hline 5 & & $.6392+/-.0852$ \\
\hline 6 & & $.8750+/-.0546$ \\
\hline 7 & & $.7623+/-.0438$ \\
\hline 8 & & $.7352+/-.0500$ \\
\hline
\end{tabular}

TABLE IV. RESULT OF LEFT/RIGHT CLASSIFICATION FOR PSD FEATURES

\begin{tabular}{|c|c|c|}
\hline $\begin{array}{l}\text { SUBJECT } \\
\text { ID }\end{array}$ & FEATURES & $\begin{array}{c}\text { CLASSIFICATION ACCURACY } \\
\text { (RSVM) }\end{array}$ \\
\hline 1 & \multirow{8}{*}{$\begin{array}{c}\text { PSD } \\
\text { (Alpha \& } \\
\text { Beta } \\
\text { Band) }\end{array}$} & $.7744+/-.0389$ \\
\hline 2 & & $.8503+/-.0671$ \\
\hline 3 & & $.8024+/-.0747$ \\
\hline 4 & & $.7147+/-.1170$ \\
\hline 5 & & $.6411+/-.0490$ \\
\hline 6 & & $.8735+/-.0853$ \\
\hline 7 & & $.7516+/-.0543$ \\
\hline 8 & & $.8047+/-.0302$ \\
\hline
\end{tabular}

TABLE V. RESULT OF ELBOW/FINGER/SHOULDER CLASSIFICATION FOR WAVELET FEATURES

\begin{tabular}{|c|c|c|c|c|}
\hline \multirow{2}{*}{$\begin{array}{c}\text { SUBJECT } \\
\text { ID }\end{array}$} & \multirow{2}{*}{ FEATURES } & \multicolumn{3}{|c|}{ CLASSIFICATION ACCURACY } \\
\hline & & RSVM-E & RSVM-F & RSVM-S \\
\hline 1 & \multirow{8}{*}{$\begin{array}{c}\text { Wavelet } \\
\text { (D3 \& D4 } \\
\text { Coeff.) }\end{array}$} & $.6508+/-.0503$ & $.6368+/-.0548$ & $.5753+/-.0990$ \\
\hline 2 & & $.6619+/-.0596$ & $.6383+/-.0269$ & $.6999+/-.0555$ \\
\hline 3 & & $.7424+/-.0718$ & $.5025+/-.0668$ & $.6123+/-.0603$ \\
\hline 4 & & $.7301+/-.0702$ & $.6329+/-.0539$ & $.6370+/-.0948$ \\
\hline 5 & & $.6864+/-.0272$ & $.5923+/-.0884$ & $.7212+/-.0914$ \\
\hline 6 & & $.6044+/-.0985$ & $.6936+/-.1138$ & $.6833+/-.0157$ \\
\hline 7 & & $.6891+/-.0628$ & $.5968+/-.0363$ & $.6704+/-.0344$ \\
\hline 8 & & $.8047+/-.0302$ & $.6137+/-.0529$ & $.6272+/-.0633$ \\
\hline
\end{tabular}

TABLE VI. RESULT OF ELBOW/FINGER/SHOULDER CLASSIFICATION FOR PSD FEATURES

\begin{tabular}{|c|c|c|c|c|}
\hline \multirow{2}{*}{$\begin{array}{c}\text { SUBJECT } \\
\text { ID }\end{array}$} & \multirow{2}{*}{ FEATURES } & \multicolumn{3}{|c|}{ CLASSIFICATION ACCURACY } \\
\hline & & RSVM-E & RSVM-F & RSVM-S \\
\hline 1 & \multirow{8}{*}{$\begin{array}{c}\text { PSD } \\
\text { (Alpha \& } \\
\text { Beta } \\
\text { Band) }\end{array}$} & $.6237+/-.0568$ & $.6701+/-.0626$ & $.6913+/-.0582$ \\
\hline 2 & & $.7574+/-.0490$ & $.9326+/-.0307$ & $.8112+/-.0679$ \\
\hline 3 & & $.6560+/-.0747$ & $.5682+/-.0983$ & $.6786+/-.0713$ \\
\hline 4 & & $.5869+/-.0781$ & $.6653+/-.0339$ & $.6980+/-.0639$ \\
\hline 5 & & $.6696+/-.0371$ & $.6212+/-.0491$ & $.7092+/-.0468$ \\
\hline 6 & & $.8011+/-.0658$ & $.5623+/-.0975$ & $.7000+/-.0650$ \\
\hline 7 & & $.6566+/-.0314$ & $.5864+/-.0919$ & $.6736+/-.0316$ \\
\hline 8 & & $.6429+/-.0722$ & $.6256+/-.0626$ & $.6477+/-.0804$ \\
\hline
\end{tabular}

For the multi-class classification, i.e., Finger-Elbow- Shoulder classification the maximum classification accuracy of $80.11 \%$ for elbow, $93.26 \%$ for finger and $81.12 \%$ for shoulder is obtained using the features obtained from power spectral density for RSVM classifier. While taking the wavelet coefficient as feature set, the maximum classification accuracy of $74.24 \%$ for RSVM classifier, as shown in Tables III to VI. All the programming was done in 'offline' mode using MATLAB environment.

\section{B. Control of the Khepera Mobile Robot-II}

Here we have used Khepera Mobile Robot-II for our demonstrations. It is equipped with 8 sensors and extensive motor control and can be used to execute a variety of movements. The motor controller can be used in two control modes: the speed and the position modes. The active control mode is set according to the kind of command received. If the controller receives a speed control command, it switches to the speed mode. If the controller receives a position control command, the control mode is automatically switched to the position mode. Different control parameters $\left(\mathrm{K}_{\mathrm{p}}, \mathrm{K}_{\mathrm{i}}\right.$ and $\left.\mathrm{K}_{\mathrm{d}}\right)$ can be set for each of the two control modes.

The commands explained in the previous section are tested on a sample classified EEG data. The hand movements detected and the corresponding control signals are given in Table VII. The actual path traced out by the robot is shown in the following snapshots (Figures 3-8).

TABLE VII. SEQUENCE OF CONTROL SIGNALS

\begin{tabular}{|c|c|c|}
\hline TIME & DETECTED HAND \\
POINTS & GESTURES & $\begin{array}{c}\text { CORRESPONDING } \\
\text { MOVEMENTS }\end{array}$ \\
\hline 1 & left elbow & move left \\
\hline 2 & left elbow & move left \\
\hline 3 & right elbow & move right \\
\hline 4 & left finger & move backward \\
\hline 5 & right shoulder & rotate clockwise \\
\hline
\end{tabular}

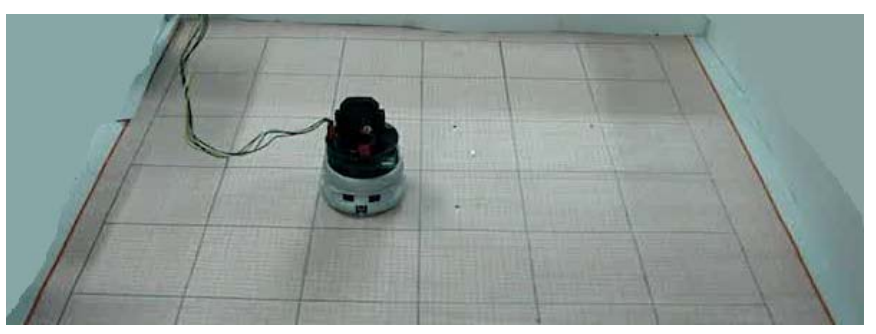

Figure 3. Initial position of the Khepera

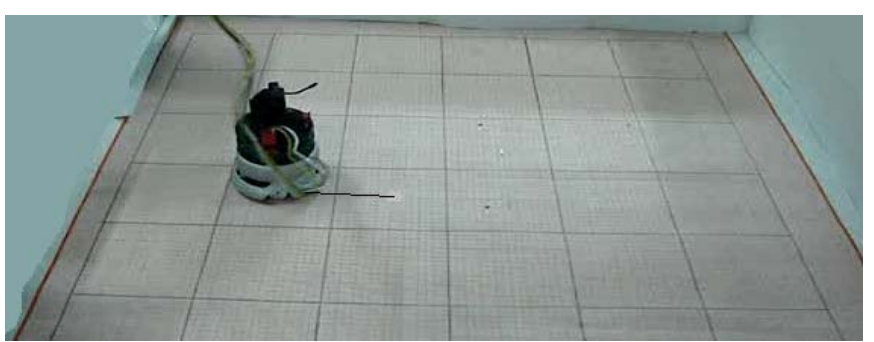

Figure 4. Motion to the left after $1^{\text {st }}$ time point 


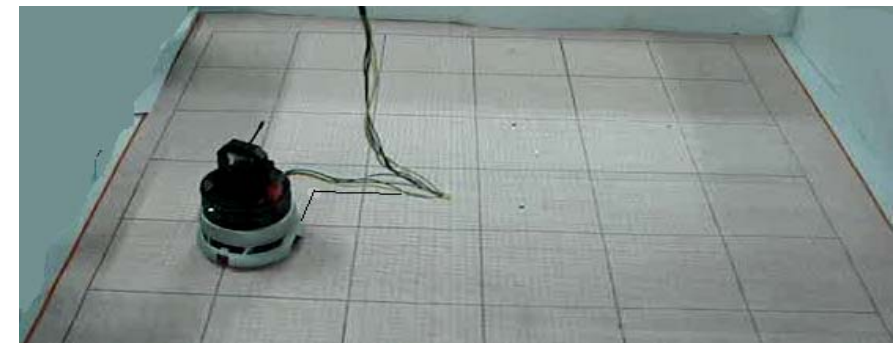

Figure 5. Motion to the left again after $2^{\text {nd }}$ time point

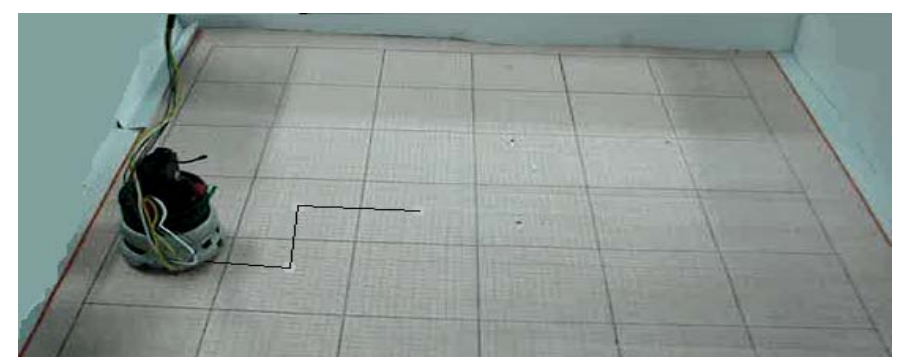

Figure 6. Motion to the right after $3^{\text {rd }}$ time point

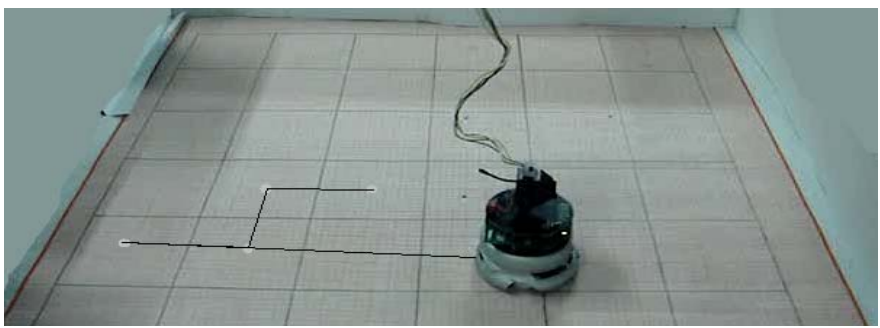

Figure 7. Backward motion after $4^{\text {th }}$ time point

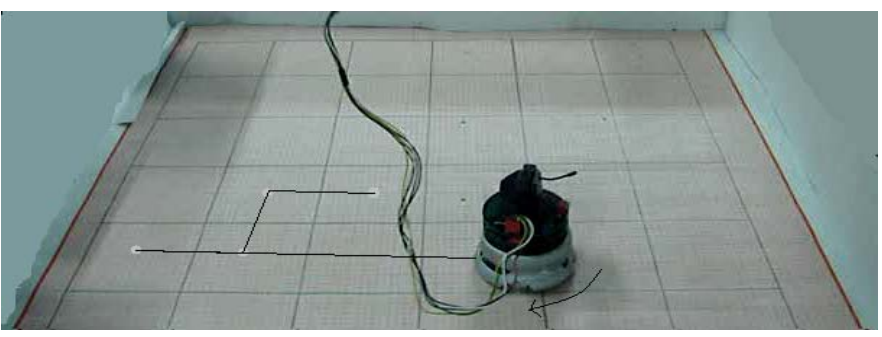

Figure 8. Clockwise rotation after $5^{\text {th }}$ time point

\section{CONCLUSIONS}

This work successfully classifies elbow-finger-shoulder movements of right and left arms with satisfactory accuracy. Experimental results showed that kernelized SVM (RBFbased) showed a superior classification result for multi-class classification. Future research work will involve improvement of the accuracy of the classifiers. In this work we have classified the data, generated the control signals and carried out the experiments separately using offline data. Obviously the next step is to implement the scheme online in real time. Thus, optimizing feature selection, extraction and classification methodologies to be implemented in online classification of EEG data for BCI research is very important for further progress in this field.

\section{REFERENCES}

[1] BCI-Robot-Therapy, http://www.healthxchange.com.sg/ News/ Pages/ BCI-Robot-Therapy.aspx

[2] Lebedev M.A., Nicoleis, "Brain-machine interface: Past, present and future", Trends Neurosci. Vol. 29(9), September 2006, pp.536-546

[3] Schloegl A., "Dynamic spectral analysis based on an autoregressive model with time-varying coefficients", IEEE-EMBC and CMBEC, 1995

[4] Darvishi S., Al-Ani A. "Brain-computer interface analysis using continuous wavelet transform and adaptive neuro-fuzzy classifier", Proc. $29^{\text {th }}$ Int. Annu. Conf. IEEE Eng. Med. Biol. Soc., August 2007, pp. 3220-3223

[5] Xu Q., Zhou H., Wang Y., Huang J. "Fuzzy support vector machine for classification of EEG signals using wavelet based features." Medical Engineering \& Physics 31, 2009, pp. 858-865

[6] Herman P., Prasad G., McGinnity T.M., Coyle D. "Comparative analysis of spectral approaches to feature extraction for EEG-based motor imagery classification." IEEE Trans. Neural sys. Rehab eng. 16(4), August 2008, pp. 317-326

[7] Gunn Steve R. "Support Vector Machines For Classification and Regression", Technical report, University of Southampton, May 1998.

[8] Burges Christopher J.C., "A Tutorial on Support Vector Machines for Pattern Recognition", Kluwer Academic Publishers, Boston, pp- 1-43

[9] V. Vapnik. "The Nature of Statistical Learning Theory". SpringerVerlag, New York, NY, 1995.

[10] Schwartz A.B., Cui X.T., Weber D.J., Moran D.W. "Brain Controlled Interfaces: Movement Restoration using Neural Prosthetics." Neuron vol.52, October 2006, pp. 205-220

[11] Pregeiizer M., Pfurtschcller G. "Distinction Sensitive Learning Vector Quantization (Dslvq) - Application as a Classifier based Feature Selection Method for a Brain Computer Interface"

[12] D.G. Childers, ed., Modern Spectrum Analysis, New York: IEEE Press, 1978

[13] Pfurscheller G., Neuper C., Schlogl A., Lugger K. "Separability of EEG signals recorded during right and left motor imagery using Adaptive Autoregressive Parameters." IEEE transaction on rehabilitation engineering 6 (3), September 1998, 316-325

[14] Cososchi S., Strungaru R., Ungureanu A., Ungureanu M., “ EEG feature extraction for motor imagery", Proc. of IEEE Engineering in Medicine and Biology Society, August 2006, 1142-1146

[15] Yom-Tov E., Inbar G.F., "Feature selection for the classification of movements from single movement-related potentials", IEEE Trans. Neural Syst. Rehab. Eng. 10, 2002, 170-178

[16] Zhendong Mu Dan Xiao, Jianfeng Hu. "Classification of motor imagery EEG signals based on STFTs"

[17] Vidaurre C., Kramer N., Blankertz B., Schlogl A. "Time Domain Parameters as a feature for EEG-based Brain Computer Interface." Neural Networks 22, 2009, 1313-1319

[18] Mahmoudi B., Erfanian A. "Single channel EEG based prosthetic hand grasp control for amputee subjects." Proceedings of the Second Joint EMBS/BMES Conference, Houston, Texas, USA, October 2002

[19] Rezaei S., Tavakolian K., Nasrabadi A. M., and Setarehdan, S. K. "Different classification techniques considering brain computer interface applications," J. Neural. Eng. 3(2), Jun 2006, 139-144

[20] Flotzinger D., Pfurtscheller G., Neuper C., Mohl W, Berger H, "Classification of non-averaged EEG data by learning vector quantization and the impact of signal processing"

[21] Thulasidas M., Guan C., and Wu J., "Robust classification of EEG signal for brain computer interface," IEEE Trans. Neural Syst. Rehab. Eng. 14(1), Mar 2006, 24-29

[22] Huang R. S., Kuo C. J., Tsai L.-L., and Chen O. T. C., "EEG pattern recognition-arousal states detection and classification," Proc. IEEE Int. Conf. Neural Netw. 2, June 1996, 641-646. 